\title{
If Entropy of Universe Is Entanglement Entropy, Is Entropy Non Zero No Matter How Small Initial Radius Is?
}

\author{
A.Beckwith \\ Chongqing University, Department of Physics, Chongqing, PRC, 400044 \\ *Corresponding Author: abeckwith@uh.edu
}

Copyright (C) 2014 Horizon Research Publishing All rights reserved.

\begin{abstract}
When initial radius R approaches zero if we use Stoica actually derived Einstein equations in a formalism which remove the big bang singularity pathology, then the reason for Planck length no longer holds. The implications of the initial radius approaching zero are the first part of this manuscript. Then the resolution is alluded to by work from Muller and Lousto, as to entanglement entropy $S_{\text {entropy }}=\frac{.3 r_{H}^{2}}{a^{2}}$ implications of entanglement entropy. We present entanglement entropy in the early universe with a steadily shrinking scale factor, due to work from Muller and Lousto, and show that there are consequences due to initial entanged entropy for a time dependent horizon radius in cosmology, with for flat space conditions $\mathrm{r}(\mathrm{H})=$ conformal time. In the case of a curved, but not flat space version of entropy, we look at vacuum energy as proportional to the inverse of scale factor squared times the inverse of initial entropy, effectively when there is no initial time except with $r_{H}=\eta$ in line with the conformal time $\eta$ being almost zero. . The consequences for this initial entropy being entangled are elaborated in this manuscript. No matter how small the initial radial length gets, then for initial cosmological entropy if it is entanglement entropy, initial cosmological entropy will not go to zero.
\end{abstract}

Keywords Entanglement Entropy, Vacuum Energy

\section{Introduction}

What happens to the topological entanglement entropy of a system, when it is driven out of its ground state by increasing the temperature? We ask this question because if the universe started as an initially cold universe, we have an initial cosmological "constant" which if it varies by temperature, could be initially set equal to zero, and which rises in value dramatically with increasing temperature. I.e. from a cold to a "hot" universe of up to
$10^{19} \mathrm{GeV} \sim 10^{32} \mathrm{Kelvin}$. The dramatic increase in temperature from a very low value to $10^{19} \mathrm{GeV} \sim 10^{32} \mathrm{Kelvin}$ would be in tandem with conditions in which Theorem 6.1.2 [1] would be when the initial conditions of the cosmos changed, no longer held. Note that we are assuming a temperature dependent cosmological 'constant' parameter, $\Lambda$, which we could write as[2] '

$$
\Lambda=\Lambda_{\text {start-value }} T^{\beta}
$$

If Eq. (1) were about zero for an ultra low temperature, we would approach having Theorem 6.1 .2 of [1]with the cosmological parameter less than or equal to zero. This is different from having a very low, constant $\Lambda_{\text {start-value }}$ and $\beta$ equal to zero, which would correspond to a uniform unchanging cosmological constant, never zero which would be with regards to the present era, very tiny and almost zero, although sufficient to initiate a deviation from Theorem 6.1.2 [1].

\section{Looking at the First Singularity Theorem and How it Could Fail, and Consequences For Entropy}

We restate at what is given by [1] as to how to state the fundamental singularity theorem

Theorem 6.1.2 (Irrotational Geodestic singularities) If $\Lambda \leq 0, \quad \rho+3 p \geq 0$, and $\rho+p>0$ in a fluid flow for which $\dot{u}=0, \omega=0$ and $H_{0}>0$ at some time $s_{0}$, then a spacetime singularity, where either $\ell(\tau) \rightarrow 0$ or $\sigma \rightarrow \infty$, occurs at a finite proper time $\tau_{0} \leq H_{0}$ before $S_{0}$.

If there is a non zero initial energy for the universe, a supposition which is counter to ADM theory as seen in [6], then the supposition by Kauffman [7] is supportable with evidence. I.e. then if there is a non zero initial energy, is this in any way counter to Theorem 6.1 above? We, keep in mind that. $\ell(\tau) \rightarrow 0$ is in reference to a scale factor, as written by $[1]$, vanishing. 


\subsection{Beckwith's Analysis of what Themerem 6.1.2 Means and the Implications for Entropy}

We find that if there is a Friedman equation interpretation of the initial singularity, that the scale factor may be interpreted as follows, namely start off with $\ell(\tau)=a(\tau)=a_{\text {initial }} e^{\tilde{H} \tau}$ with $\tilde{H}$ an initial huge Hubble parameter[1]

$$
\begin{gathered}
3 \ddot{a} / a=-4 \pi G \cdot(\rho+3 p)+\Lambda \Rightarrow \dot{a}^{2}-8 \pi G \rho a^{2}-\Lambda a^{2}-\text { const }=0 \\
a_{\text {initist }}^{2}=\text { const } /\left[\tilde{H}^{2}-[8 \pi G \rho+\Lambda]\right] \Rightarrow \\
a_{\text {isims } !}=\sqrt{\text { const } /\left[\tilde{H}^{2}-[8 \pi G \rho+\Lambda]\right]}
\end{gathered}
$$

Aside from the Planck "constant" being less than or equal to zero, one would also need to consider a temperature dependent Hubble parameter, with for most cases an inequality for the denominator of Eq. (3) we could read as (assuming $100<g_{*}(T)<1000$, i.e. high but not infinite degrees of freedom)

$$
(1.7)^{2} g_{*}(T) \cdot \frac{T^{4}}{M_{p}^{2}}>>8 \pi G \rho_{\text {initial }}+\Lambda_{\text {start-Value }} T^{\beta}
$$

We argue that in many cases we will be looking at nonzero values of Eq. (3) above. And we shall now see what the implications are for initial entanglement entropy, which we will define in the next section. Using [3] definition of entropy, assuming a Roberson-Walker almost flat space treatment of space time even initially we could write as given below, with a time dependent square of the Horizon radius $r_{H}$. So that if we have an initial value of the Horizon radius as given by $r_{H}=\eta, \eta$ as conformal time, initially, then by [3]

$$
S_{\text {entropy }}=\frac{.3 r_{H}^{2}}{a^{2}}
$$

We then could write Eq. (4) as initial entanglement entropy, with $\eta$ a piece of information transferred from a prior universe to our own present universe. To do this we will utilize cyclic conformal cosmology [4], as a method of specifying what $\eta$ is transferred from a prior universe to complete what can be said for initial entropy in Eq. (5) above.

\subsection{Beckwith's Analysis of what Theorem 6.1. Means Due to Entanglement Transfer of Information from A Prior Universe Leading to an Initial Value of $r_{H}=\eta$}

What we are assuming is that there is a nonzero amount of information transferred from a prior to the present universe for being able to have as a set value initially $r_{H}=\eta$. Our way of obtaining nonzero amounts of transferred information via entanglement much as has been done in EPR quantum entanglement as applied to a cyclic universe construction [4].

\section{Examining Possibly Using Cyclic Conformal Cosmology as Given by Penrose to Lead to a Prior Universe Contributing to Initial Entropy as Given by Eq. (4) above.}

Penrose [4]suggested as to recycling from a prior universe to our initial configuration for the present universe gives the following as an invariant quantity, from a prior to a present universe

$$
\Pi=\frac{d \Omega}{\Omega^{2}-1} \underset{\Omega \underset{C C C}{\longrightarrow} \Omega^{-1}}{\longrightarrow} \operatorname{SAME} \frac{d \Omega}{\Omega^{2}-1}
$$

Here, we have that there is a cyclic conformal mapping, i.e. from large final state, to small initial state

$$
\Omega \underset{C C C}{\longrightarrow} \Omega^{-1}
$$

So that information provided by $\hat{g}=\Omega^{2} g$ goes from a small to a large metric and then is recycled into a new $g$ ( cosmological metric) for each new recycled universe. Key to doing this would be an EPR entanglement transfer, of invariant information, so that we have a set value of $r_{H}=\eta$ [4].

We suggest that as part of the invariance given by Eq.(5) that there is EPR entanglement transfer of information so as to permit having $r_{H}=\eta$ fixed by quantum entanglement from a prior to the present universe, as part of the information transfer implied by Eq. (5) above., i.e. we have that there exists a gravitational source, $\mathrm{E}$, which is defined for a given energy tensor $\mathrm{T}$ so that it does not change from cycle to cycle, i.e that as part of the EPR transfer of information[5] for a value of $r_{H}=\eta$, we have a re set of the gravitational source information $\mathrm{E}$ from cosmological cycle to another cosmological cycle as defined by $\hat{g}=\Omega^{2} g$ being re set, by $\Omega \underset{C C C}{\longrightarrow} \Omega^{-1}$ through each cosmological cycle put into the gravitational source information, E, as given below. Here, we have that $\hat{g}=\Omega^{2} g$ grows from a very small term to a very large term, and that the magnitude of the large value of $\hat{g}=\Omega^{2} g$ is re set via $\Omega \underset{C C C}{\longrightarrow} \Omega^{-1}$ at the start of each cosmological cycle [4].

$$
E=8 \pi T+g \Lambda
$$




\section{Conclusion. Non Zero Entangled Entropy Provided by EPR Transfer of Information for Value of Information for $r_{H}=\eta$, from Prior to Present Universe and Finite Value of L.H.S. of Eq. (3)}

All what has been written in this article depends upon confirming Eq. (3) as well as deciphering experimental signatures for the gravitational source expression, Eq. (7). Our hypothesis about the invariance of Eq. (5) from cycle to cycle, combined with a reset of initial information as contained in a $r_{H}=\eta$ fixed by quantum entanglement from a prior to the present universe, as part of the information transfer implied by Eq. (5) above, is crucial to the conclusion we have that we do not want a singularity at the beginning, leading to a tiny but non zero $a$ (scale factor), combined with a tiny but non zero initial $r_{H}=\eta$ transmitted via EPR style entanglement from a prior to a present universe at the same time, we have $\Omega \underset{C C C}{\longrightarrow} \Omega^{-1}$

put into metric $\hat{g}=\Omega^{2} g$ [4] at the start of cosmological expansion. Doing so will, we estimate lead to setting, due to entangled information creating $r_{H}=\eta$ initially so that, no matter how small $a$ (scale factor) is, we will be able to write a non zero entropy [3].

$$
S_{\text {initial-entropy }}=\left.\frac{.3 r_{H}^{2}}{a^{2}}\right|_{\text {initial-values }} \propto 10^{5}
$$

We expect that any confirmation of Eq. (9) preferably via Eq.(8) and perhaps traces in the CMBR will also look at what happens if we have, instead of Eq.(1) above an effective potential as given by

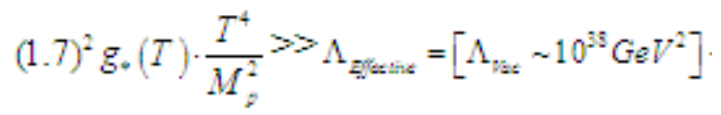

$$
\begin{aligned}
& +\left[\Lambda_{S D^{\sim}} \sim \text { const } \mathrm{T}^{|\xi|}\right] \sim 10^{38} \mathrm{GeV}^{2} \text { (Plandk - era) }
\end{aligned}
$$

Confirming Eq. (10) will be what we will look at next. It means taking Brane theory seriously as a way to get an effective cosmological constant, in Eq.(10) ,to approach todays ultra low energy value. [8] as a reference gives a specific entry as to what could be a way to nullify the usual situation which is represented by [9]. I.e. massive gravitons usually are expected to change the bending of light by $3 / 4$ its GR predicted value. Should [8] be confirmed as a reality, if gravitons have a mass, then it means that massive gravity is fully commensurate with Eq.(10) above which will perhaps then lead to investigation of a prediction as to 'fat gravitons' contributing to dark energy and the speed up of the universe
[10] . The same suggestion by [10] was also brought up by the author in [11] earlier. That will be an exciting datum to investigate if true. But it would have major consequences for Eq.(10) above as well as the implications as of Eq.(9). In addition it would also confirm what the author wrote up in [12] as far as cyclic universes and information transfer [12]. The author's expectation is that confirmation of Eq. (10) plus some of the work done in [12] will be crucial in determination as to what would be transferred via entropy and information as far as seeding of the cosmological parameters from cycle to cycle.

\section{REFERENCES}

[1] G. Ellis, R. Maartens, and M.A. H. MacCallum;'Relativistic Cosmology", Cambridge University press,2012,Cambridge, U.K

[2] D. K. Park, H. Kim, and S. Tamarayan, "Nonvanishing Cosmological Constant of Flat Universe in Brane world Scenarios," Phys.Lett. B535 (2002) pp. 5-10

[3] R. Muller and C.O. Lousto,'Enganglement entropy in curved spacetimes with event horizons",arXiv:gr-qc/9504049 v1

[4] R. Penrose, Cycles of Time, The Bodley Head, 2010, London, UK

[5] Eduardo Martin-Martinez, Nicolas C. Menicucci, "Cosmological Quantum Entanglement", http://arxiv.org/abs/1204.4918

[6] Edward Kolb , Michael Turner, "The Early Universe", Westview Press (February 21, 1994)

[7] Steven Kauffmann, “ A Self Gravitational Upper bound On Localized Energy Including that of Virtual Particles and Quantum Fields, which Yield a Passable Dark Energy Density Estimate", http://arxiv.org/abs/1212.0426

[8] C. Csaki, J. Erlich, T. Hollowood, " Graviton propagators, Brane Bending and the Bending of Light in Theories with Quasi-Localized Gravity", Phys.Lett.B481:107-113,2000, http://arxiv.org/abs/hep-th/0003020

[9] P. Tinyakov, "Giving Mass to the Graviton", pp 471-499 of "Particle physics and cosmology, the Fabric of spacetime" by Les Houches, Session LXXXVI,2006, edited by F. Bernardeau, C. Grojean, and J. Dalibard, and published by Elsevier , 2007 Oxford UK

[10] http://www.nature.com/news/fat-gravity-particle-gives-clues -to-dark-energy-1.13707

[11] A.Beckwith, http://journalofcosmology.com/BeckwithGraviton.pdf

[12] A. Beckwith," How to have a transfer of information from a prior to a present universe in lieu of information theory", Volume 3, issue 1 of JAM, October 2013, http://cirworld.com/index.php/JAM/article/view/2475/pdf_1 7 